\title{
Apoptosis: Cell death defined by caspase activation
}

\author{
Dear Editor, \\ 'The term apoptosis is proposed for a hitherto little recognized \\ mechanism of controlled cell deletion, which appears to play a \\ complementary but opposite role to mitosis in the regulation \\ of animal cell populations.'
}

Kerr, Wyllie and Currie, Br. J. Cancer, 26, p 239-257, 1972.

'If for convenience we accept the word, we must be careful not to use it too glibly.'

The Lancet, 11, p 1011-2, 1972.

In 1972, Kerr et al ${ }^{1}$ recognized that developmental, physiologic and some pathologic forms of cell death shared common features that were distinct from necrosis. The authors proposed the term 'apoptosis' to refer to this morphologic form of cell death. During the intervening period, there has been an explosive growth of research in this area, with over 8000 papers published in the past year alone. These studies have addressed cell death in a very broad spectrum of cell types and physiologic and pathologic conditions. The accumulation of data shows that the constellation of morphologic features identified as apoptosis are common but not uniform. Each of the characteristics, i.e. formation of nuclear and plasma membrane blebs, condensation of chromatin, loss of cell volume, detachment of cells from basement membranes, when considered alone, does not provide an unambiguous definition of apoptosis. There are time-dependent changes, cell-specific characteristics, and normal physiologic processes that introduce sufficient variation in morphology so that no single morphologic trait can define this type of cell death. Accordingly, it is highly desirable to have a definition of apoptosis based upon the underlying mechanism. Efforts to understand the mechanistic basis of apoptosis have yielded compelling evidence that activation of a caspase cascade provides a common biochemical pathway that explains both the morphologic features and the irreversible commitment point for this type of cell death. The purpose of this communication is to propose that apoptosis be redefined in biochemical terms as a caspase-mediated cell death with associated apoptotic morphology, i.e. cytoplasmic and nuclear condensation, chromatin cleavage and exposure of surface markers targeting cells for phagocytosis.

Multicellular organisms (animals) share a common mechanism to control cell populations that has been conserved during evolution. The genetic basis of programmed cell death in Caenorhabditis elegans, was elucidated over a decade ago., ${ }^{2,3}$ This involves a cell death protease (CED-3) which is associated with activating (CED4) and inhibiting (CED-9) proteins. ${ }^{4}$ Homologous proteins have been found in organisms throughout the animal kingdom, including insects, amphibians, rodents and primates. ${ }^{5}$ The homologues of CED-3 are termed caspases, or cysteine-dependent aspartate-specific proteases. ${ }^{6}$ Overexpression of these proteins can result in cell death with apoptotic morphology. However, not all of these caspases normally function in cell death. A recent classification divided caspases into two groups, one involved in cytokine processing (caspase-1-like proteases) and the other playing a role in cell death. This latter group can be further subdivided according to function as activators or executioners of cell death ${ }^{7}$ and represent an evolution of this common mechanism for control of cell populations.

Although full details and variations of the pathway have not been completely delineated, cell death in a variety of models involves activation of caspase(s). In surfacereceptor-mediated killing, activation of caspase- 8 is an early event that initiates a caspase cascade and results in cleavage of different proteins located throughout the cell. ${ }^{8,9}$ In cytotoxic $\mathrm{T}$ lymphocyte-mediated killing, granzyme B activates caspases in target cells which results in cleavage of both nuclear and cytoplasmic proteins. ${ }^{10}$ In cells exposed to ionizing radiation or other DNA-damaging agents where p53 is activated, cell death is mediated by caspases. ${ }^{11}$ In stress-activated cell death induced by heat shock, cold shock, viral infection, bacterial infection, or growth factor withdrawal, caspase activation is a common feature of cell death (for review see reference 5). In steroidmediated cell killing, caspases are also activated. ${ }^{12}$ In mitochondria-mediated cell death, cytochrome $c$ release leads to a caspase activity which is dependent upon a CED-4 homologue, Apaf- $1 .{ }^{13}$ In all of these cases, small peptide-based caspase inhibitors delay or block appearance of apoptotic morphology and cell death. Moreover, the recent development of several caspase or Apaf-1 knockout animals, has provided more definitive support for the importance of these proteases in apoptosis. Thus, abundant evidence leads to the conclusion that caspase activation is a common pathway for the execution of this type of cell death.

The proteolytic activity of caspases provides a molecular basis for apoptotic morphology. Structural components such as nuclear lamins and cytoskeletal proteins that bind to the plasma membrane are cleaved by caspases, preceding nuclear condensation and membrane blebbing. ${ }^{5}$ Caspase-dependent cleavage of ICAD (inhibitor of the caspase-activated $D N$ Nase) allows activation of CAD and cleavage of DNA into characteristic oligonucleosomallength fragments. ${ }^{14}$ Exposure of phosphatidylserine on the cell surface, which is a marker for phagocytosis, is blocked by caspase inhibitors. ${ }^{15}$

Similarly, functional changes associated with controlled cell deletion are also linked to the cleavage of protein substrates for caspases (for review see reference 16). In 
particular, caspases cleave and activate different proteins (kinases) important for the signal transduction pathways controlling cell growth and function. Caspases cleave negative regulators of apoptosis and either inactivate them or produce fragments that promote cell death, e.g., Bcl-2 and $\mathrm{Bcl}-\mathrm{x}_{\mathrm{L}}$. Several enzymes involved in maintaining DNA stability and repair, including poly(ADP-ribose) polymerase, DNA - PK and DNA replication factor 140, are cleaved and inactivated by caspases.

Further evidence supporting the definition of apoptosis in terms of caspase activation comes from studies of chemical-induced cell death. In many cases, there is a shift from apoptosis to necrosis with increasing concentration of a toxic compound. This shift in morphology can occur when there is inhibition of caspases ${ }^{17}$ and/or a direct loss of energy metabolism or osmotic stability. ${ }^{18}$

The starting point in the definition of apoptosis was recognition of a common morphologic process despite an underlying heterogeneity in cell phenotype. The evidence is now clear that caspase activation provides a common biochemical basis for this morphology despite an underlying heterogeneity in different cell types in its control and activation.

Identification of defective components in cancer and degenerative diseases as well as viral mechanisms to subvert caspase activation underscore the central importance of this pathway in the regulation of cell populations. $^{19}$

There are now several examples of caspase activation without cell death ${ }^{20}$ and cell death without caspase activation. ${ }^{21,22}$ As indicated above, caspases have other cell functions (e.g., cytokine processing) and thus, activation of caspases is a necessary but not a sufficient indicator for apoptosis. Similarly, there are caspaseindependent mechanisms that can cause cell blebbing, DNA fragmentation and phosphatidylserine exposure on the cell surface. However, experiments showing apoptotic morphology without detectable caspase activity or lack of protection with caspase inhibitors do not provide conclusive evidence that caspase-independent apoptosis exists. Indeed, if a caspase-independent biochemical mechanism for controlled cell death is identified, it should certainly receive an alternate terminology to enhance precision in communication. Defining apoptosis as a cell death process mediated by caspases allows other forms of cell death, mediated by other mechanisms, to be clearly distinguished. Thus these processes should be simply referred to as 'cell death' until the distinguishing morphologic features and underlying biochemical mechanisms are recognized.
Acceptance of caspase-dependent cell death, whether programmed, physiological, pathological or inducible in nature, as synonymous with apoptosis will allow delineation of other forms of cell death and will facilitate communications regarding these processes. We strongly urge investigators of all forms of cell death to adopt this definition.

\author{
Afshin Samali ${ }^{1}$ \\ Boris Zhivotovsky ${ }^{1}$ \\ Dean Jones ${ }^{2}$ \\ Shigekazu Nagata ${ }^{3}$ \\ Sten Orrenius ${ }^{*}, 1$ \\ ${ }^{1}$ Institute of Environmental Medicine, \\ Division of Toxicology, Karolinska Institutet, \\ S-171 77 Stockholm, Sweden \\ ${ }^{2}$ Department of Biochemistry, Emory University, \\ Atlanta, GA 30322, USA \\ ${ }^{3}$ Department of Genetics, \\ Osaka University Medical School, \\ 2-2 Yamada-oka/Suite, Osaka 565, Japan \\ *Corresponding Author, Tel: +46-8-728 7590, \\ Fax: +46-8-32 90 41, \\ e-mail: sten.orrenius@imm.ki.se
}

\section{References}

(Owing to space limitation, the authors apologize that many articles which significantly contribute to elucidation of central role of caspases in apoptosis could not be cited.)

1. Kerr JFR, Wyllie AH and Currie AR (1972) Br. J. Cancer, 26: 239-257.

2. Horvitz HR, Ellis HM and Sternberg PW (1982) Neurosci. Comment 1: 56-65

3. Hedgecock EM, Sulston JE and Thomson JN (1983) Science 220: 1277-1279

4. Metzstein MM, Stanfield GM and Horvitz HR (1998) Trends Genet. 14: 410-416

5. Cryns V and Yuan J (1998) Genes Develop. 12: 1551-1570

6. Alnemri ES et al (1996) Cell 87: 171

7. Thornberry NA and Lazebnik Y (1998) Science 281: 1312-1317

8. Boldin MP et al (1996) Cell 85: 803-815

9. Muzio M et al (1996) Cell 85: $817-827$

10. Shi L et al (1996) Proc. Natl. Acad. Sci. U.S.A. 93: 11002-11007

11. Datta R et al (1997) J. Biol. Chem. 272: 1965-1969

12. Zhivotovsky B et al (1995) Exp. Cell Res. 221: 404-412

13. Li PD et al (1997) Cell 91: 479-489

14. Enari $\mathrm{M}$ et al (1998) Nature 391: $43-50$

15. Vanags DM et al (1996) J. Biol. Chem. 271: 31075-31085

16. Stroh C and Schulze-Osthoff K (1997) Cell Death Differ. 5: 997-1000

17. Lemaire $C$ et al (1998) FEBS Lett. 425: 266-270

18. Leist M et al (1997) J. Exp. Med. 185: $1481-1486$

19. Webb SJ, Harrison DJ and Wyllie AH (1997) Adv. Pharmacol. 41: 1-34

20. William R et al (1998) J. Immunol. 161: 957-962

21. Deas O et al (1998) J. Immunol. 161: $3375-3383$

22. Kawahara A et al (1998) J. Cell Biol. 143: 1353-1360 